\title{
Error estimates for 1D asymptotic models in coaxial cables with non-homogeneous cross-section
}

\author{
Sébastien Imperiale ${ }^{1,2}$ and Patrick Joly ${ }^{2, *}$ \\ ${ }^{1}$ Department of Applied Physics and Applied Mathematics, Columbia University, \\ New York NY, 10027. \\ 2 INRIA Rocquencourt, POems, domaine de Voluceau, 78153 Le Chesnay, FRANCE
}

\begin{abstract}
This paper is the first contribution towards the rigorous justification of asymptotic 1D models for the time-domain simulation of the propagation of electromagnetic waves in coaxial cables. Our general objective is to derive error estimates between the "exact" solution of the full 3D model and the "approximate" solution of the 1D model known as the Telegraphist's equation.
\end{abstract}

AMS subject classifications: 35L05, 35A35, 73R05, 35A40

Key words: Coaxial cables, Telegraphist's equation, Error estimates, Non-homogeneous crosssection

\section{Introduction}

This work is a continuation of a previous article [4] devoted to the asymptotic modeling of electromagnetic waves propagations in a thin co-axial cable. By thin cable, we mean a 3D elongated (infinitely long in this paper) cylindrical domain whose transverse dimensions are small with respect to the considered wavelengths. By co-axial cable, we mean that each transverse cross-section of the cable is not simply connected, which is essential. Of course, as a cable is a thin structure whose transverse dimensions are much smaller than the longitudinal one, one would like to use a simplified 1D model : this is even necessary for the effective efficiency of the computational tool (one wants in particular to avoid using a 3D mesh for the thin cable). In such a situation, electrical engineers use the well-known Telegraphist's equations for "perfect" coaxial cables (homogeneous with circular cross-section) where the electric unknowns are reduced to an electric potential $V\left(x_{3}, t\right)$ and an electric current $I\left(x_{3}, t\right)$, where $x_{3}$

\footnotetext{
*Corresponding author.

Email: si2245@columbia.edu (Sébastien Imperiale), patrick.joly@inria.fr (Patrick Joly)
} 
denotes the abscissa along the cable, $t$ is time and in absence of source

$$
\left\{\begin{array}{c}
C \frac{\partial V}{\partial t}+G V-\frac{\partial I}{\partial x_{3}}=0 \\
L \frac{\partial I}{\partial t}+R I-\frac{\partial V}{\partial x_{3}}=0
\end{array}\right.
$$

where the capacitance $C$, the inductance $L$, the conductance $G$ and the resistance $R$ can be expressed it terms of the geometry of the cross-section. In [4], thanks to a formal asymptotic expansions with respect to the small parameter $\delta:=$ diameter of the cable / unit reference length, we derived a simplified 1D effective model under quite general assumptions: the cross section is heterogeneous, slowly variable in the longitudinal direction and possibly made of lossy media (i.e. with electric or magnetic conductivities). To derive this effective model, we considered a family of problems posed in domains that depend on a small geometric parameter $\delta>0$. Of course, a given cable corresponds to a given value of $\delta$ but the effective model will be constructed by an asymptotic analysis in $\delta$. The resulting model appears as an extension of the Telegraphist's equation (1.1) currently used in the engineering community [3], [8] (in particular, we show that the presence of lossy media induces the apparition of time convolution terms in the limit model). The coefficients of the homogenized model are given explicitly as the solutions of two 2D scalar elliptic problems posed in the cable cross-section. Such models can be used as an efficient tool for the time-domain numerical simulation of the propagation of electromagnetic waves in coaxial cables, which is needed in many industrial applications. In our case, we were motivated by the simulation of non-destructive testing experiments by ultra-sounds [5], where coaxial cables are used for the electric supply process for piezo-electric transducers [9].

The present paper is the first contribution towards the rigorous justification of the results of [4]. More than a simple convergence theorem, the general objective is to derive error estimates (in a sense that will be explained later) between the "exact" solution of the full 3D model and the "approximate" solution of the 1D model. We focus in this first paper on the (model) situation of a perfectly cylindrical cable (invariant under translation in the longitudinal direction) whose cross-section is heterogeneous (constitutive coefficients depend on transverse variables) but made of non-lossy media. A more general situation will be considered in a future work.

The paper is organized as follows. In section 2, we present the considered model problem and more precisely the family of $\delta$-dependent problems that we wish to analyze. In section 3, we recall the main results of [4] in the simplified situation considered in this paper. Then we give the main results of this work (theorem 3.2), that provide various error estimates under the only assumption that the data of the problem (the source terms) are adequately "well-prepared". Finally, in section 4, we give a detailed proof of theorem 3.2, that relies on appropriate vector field decompositions, energy estimates and adequate versions of Poincaré-Friedrichs inequalities (see Appendix 5). 


\section{The homogenized 1D model in non-conductive cylindrical coaxial cables}

We consider a family of problems posed in cylindrical domains (cables) that depend on a small geometric parameter $\delta>0$ measuring the transverse dimensions of the cables. The domain associated to the cables are denoted $\Omega^{\delta}$ :

$$
\Omega^{\delta}=\delta S \times \mathbb{R}
$$

where $S$ is a connected bounded and Lipschitz reference domain (of unit diameter) in $\mathbb{R}^{2}$ (see Figure 1 ). In what follows, we shall denote by $\mathbf{x}=\left(x, x_{3}\right)$ the 3D space variable where $x=\left(x_{1}, x_{2}\right)$ represents the transverse coordinates. An essential assumption is that $S$ is not simply connected:

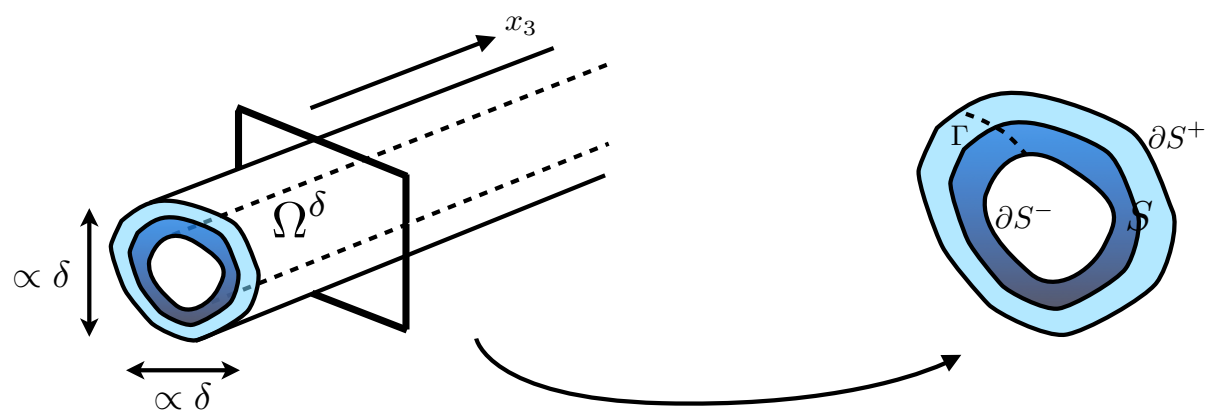

Figure 1: Geometry of the coaxial cables.

$$
S=\mathcal{O} \backslash \bar{T}, \quad \bar{T} \subset \subset \mathcal{O}
$$

where $\mathcal{O}$ and $T$ (the hole) are simply connected, Lipschitz, open sets of $\mathbb{R}^{2}$. This corresponds to the case where the cable contains only one metallic (perfectly conducting) wire. However, the extension to several holes (or several metallic wires) is rather straightforward [4]. In this case, the boundary of $S$ has two connected components, the exterior one $\left(\partial S^{+}\right)$and the interior one $\left(\partial S^{-}\right)$:

$$
\partial S^{+}:=\partial \mathcal{O}, \quad \partial S^{-}:=\partial T .
$$

To define the effective model, we shall introduce an artificial cut in the cross-section, namely a line $\Gamma$ joining $\partial S^{+}$to $\partial S^{-}$so that the domain:

$$
S^{\Gamma}:=S \backslash \Gamma,
$$

is simply connected. The family of (thin) domains $\Omega^{\delta}$ is related to the reference domain $\Omega=S \times \mathbb{R}$ by

$$
\Omega^{\delta}=\mathcal{G}_{\delta}(\Omega),
$$


with the transverse scaling transformation $\mathcal{G}_{\delta}:\left(x_{1}, x_{2}, x_{3}\right) \longrightarrow\left(\delta x_{1}, \delta x_{2}, x_{3}\right)$. Along $\partial \Omega^{\delta}$, the outward unitary normal vector $\mathbf{n}^{\delta}$ satisfies

$$
\text { a. e. } \mathcal{G}_{\delta}(\mathbf{x}) \in \partial \Omega^{\delta}, \quad \mathbf{n}^{\delta}=\left(n_{1}(\mathbf{x}), n_{2}(\mathbf{x}), 0\right)^{t}, \quad n=\left(n_{1}, n_{2}\right)^{t}: \partial S \rightarrow \mathbb{R}^{2},
$$

where $n$ is the (2D) outward unitary normal vector to $\partial S$. Next, we assume that the material properties, namely the electric permittivity $\varepsilon^{\delta}(\mathbf{x})$ and magnetic permeability $\mu^{\delta}(\mathbf{x})$, do not depend on $x_{3}$ and are obtained by a scaling in $x=\left(x_{1}, x_{2}\right)$ of fixed distributions over the reference domain $\Omega$ :

$$
\varepsilon^{\delta}=\varepsilon \circ \mathcal{G}_{\delta}^{-1}, \quad \mu^{\delta}=\mu \circ \mathcal{G}_{\delta}^{-1},
$$

and where $(\varepsilon, \mu)$ are identified to (measurable) functions defined on $S$ that satisfy the usual positivity properties:

$$
0<\varepsilon_{-} \leq \varepsilon(x) \leq \varepsilon_{+}, \quad 0<\mu_{-} \leq \mu(x) \leq \mu_{+}, \quad \text { a. e. } x \in S .
$$

The equations governing the electric field $E^{\delta}(x, t)$ and the magnetic field $H^{\delta}(x, t)$ are Maxwell's equations ( $\nabla \times$ denoting the usual 3D curl operator)

$$
\left\{\begin{array}{l}
\varepsilon^{\delta} \frac{\partial E^{\delta}}{\partial t}-\nabla \times H^{\delta}=J^{\delta}, \quad \text { in } \Omega^{\delta}, \quad t>0 \\
\mu^{\delta} \frac{\partial H^{\delta}}{\partial t}+\nabla \times E^{\delta}=0, \quad \text { in } \Omega^{\delta}, \quad t>0
\end{array}\right.
$$

with perfectly conducting boundary conditions

$$
E^{\delta} \times \mathbf{n}^{\delta}=0 \quad \text { on } \partial \Omega^{\delta}, \quad t>0,
$$

the system being considered at rest at $t=0$ :

$$
E^{\delta}(\mathbf{x}, 0)=H^{\delta}(\mathbf{x}, 0)=0, \quad \text { a. e. } \mathbf{x} \in \Omega^{\delta} .
$$

To proceed in our analysis we shall assume that the source term, namely the current density $J^{\delta}(\mathbf{x}, t)$, has no longitudinal component, is divergence free, vanishes at time $t=0$ and is obtained by scaling in $\left(x_{1}, x_{2}\right)$ of a fixed current density in $\Omega$ (this corresponds to what we call well-prepared data):

$$
J^{\delta}=J \circ \mathcal{G}_{\delta}^{-1}, \quad J=\left(J_{1}, J_{2}, 0\right)^{t}, \quad \frac{\partial J_{1}}{\partial x_{1}}+\frac{\partial J_{2}}{\partial x_{2}}=0, \quad J(\mathbf{x}, 0)=0, \quad \text { a. e. } \mathbf{x} \in \Omega .
$$

For the analysis, it appears judicious to introduce the tangential components

$$
E_{T}^{\delta}=\left(E_{1}^{\delta}, E_{2}^{\delta}\right)^{t}, \quad H_{T}^{\delta}=\left(H_{1}^{\delta}, H_{2}^{\delta}\right)^{t},
$$

of the electric and magnetic fields as well as the longitudinal components of these fields: $E_{3}^{\delta}$ and $H_{3}^{\delta}$. We can rewrite the equations (2.5) with these new unknowns, using 
the following notations: for all scalar functions $u$ and 2D transverse vector fields $v$ and $w$ with two components $v_{1}$ and $v_{2}$ or $w_{1}$ and $w_{2}$, we define:

$$
\nabla u \equiv\left(\frac{\partial u}{\partial x_{1}}, \frac{\partial u}{\partial x_{2}}\right)^{t}, \overrightarrow{\operatorname{rot}} u \equiv\left(\frac{\partial u}{\partial x_{2}},-\frac{\partial u}{\partial x_{1}}\right)^{t}, \operatorname{div} v \equiv \frac{\partial v_{1}}{\partial x_{1}}+\frac{\partial v_{2}}{\partial x_{2}}, \operatorname{rot} v \equiv \frac{\partial v_{2}}{\partial x_{1}}-\frac{\partial v_{1}}{\partial x_{2}} .
$$

Moreover, for any $v=\left(v_{1}, v_{2}\right)$ and $w=\left(w_{1}, w_{2}\right)$, we shall set

$$
v \cdot w \equiv v_{1} w_{1}+v_{2} w_{2}, \quad v \times w \equiv v_{1} w_{2}-v_{2} w_{1}, \quad \mathbf{e}_{3} \times v \equiv\left(-v_{2}, v_{1}\right)^{t} .
$$

We will also used the following properties,

$$
\overrightarrow{\operatorname{rot}} u \cdot \nabla \widetilde{u}=-\overrightarrow{\operatorname{rot}} \tilde{u} \cdot \nabla u, \quad \mathbf{e}_{3} \times \nabla u=-\overrightarrow{\operatorname{rot}} u, \quad \nabla u \times v=-v \cdot \overrightarrow{\operatorname{rot}} u .
$$

Remark 2.1. In the sequel, we shall denote the $L^{2}$ scalar product in a domain $D \subset \mathbb{R}^{2}$ (with $n$ the unit outgoing normal vector to $\partial D$ ) of two scalar functions $u$ and $\widetilde{u}$ in $L^{2}(D)$ or two vector fields $v$ and $\widetilde{v}$ in $L^{2}(D)^{2}$ as

$$
(u, \widetilde{u})_{L^{2}(D)}=\int_{D} u \widetilde{u} d \mathbf{x}, \quad(v, \widetilde{v})_{L^{2}(D)}=\int_{D} v \cdot \widetilde{v} d \mathbf{x}
$$

and $\|\cdot\|_{L^{2}(D)}$ this associated norm. We shall also use the following Green's formula:

$$
\langle v \times n, u\rangle_{\partial \Omega}=(v, \overrightarrow{r o t} u)_{L^{2}(\Omega)}-(\operatorname{rot} v, u)_{L^{2}(\Omega)},
$$

valid for any $(u, v) \in L^{2}(\Omega) \times L^{2}(\Omega)^{2}$ such that $\overrightarrow{r o t} u \in L^{2}(\Omega)^{2}$ and $v \in L^{2}(\Omega)$ and where $\langle\cdot, \cdot\rangle$ represents the duality product between $H^{1 / 2}(\partial \Omega)$ and $H^{-1 / 2}(\partial \Omega)$.

The equations (2.5) can be rewritten as (with $J_{S}^{\delta}=\left(J_{1}^{\delta}, J_{2}^{\delta}\right)^{t}$ )

$$
\begin{cases}\varepsilon^{\delta} \frac{\partial E_{T}^{\delta}}{\partial t}-\mathbf{e}_{3} \times \frac{\partial H_{T}^{\delta}}{\partial x_{3}}-\overrightarrow{\operatorname{rot}} H_{3}^{\delta}=J_{S}^{\delta,} & \text { in } \Omega^{\delta}, \quad t>0 \\ \varepsilon^{\delta} \frac{\partial E_{3}^{\delta}}{\partial t}-\operatorname{rot} H_{T}^{\delta}=0, & \text { in } \Omega^{\delta}, t>0 \\ \mu^{\delta} \frac{\partial H_{T}^{\delta}}{\partial t}+\mathbf{e}_{3} \times \frac{\partial E_{T}^{\delta}}{\partial x_{3}}+\overrightarrow{\operatorname{rot}} E_{3}^{\delta}=0, & \text { in } \Omega^{\delta}, t>0 \\ \mu^{\delta} \frac{\partial H_{3}^{\delta}}{\partial t}+\operatorname{rot} E_{T}^{\delta}=0, & \text { in } \Omega^{\delta}, t>0 .\end{cases}
$$

Using (2.2), the boundary conditions (2.6) become

$$
E_{T}^{\delta} \times n=0, \quad E_{3}^{\delta}=0, \quad \text { on } \partial \Omega^{\delta}, \quad t>0 .
$$

Moreover, taking the divergence of the equations (2.11) and using (2.8), we get, after time integration, the "hidden" divergence equations:

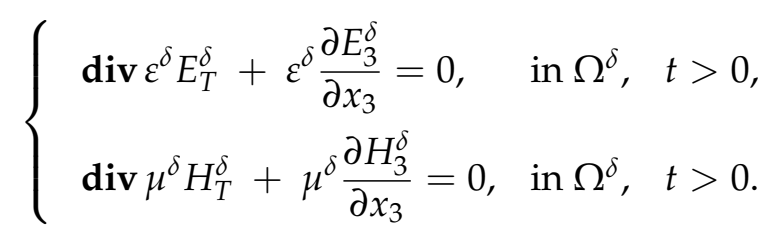


Finally, from the Maxwell's equations (2.11), the divergence equations (2.13) and the boundary conditions (2.12), it is classical to derive an additional hidden boundary condition for the magnetic field (see for instance [2]):

$$
H_{T}^{\delta} \cdot n=0, \quad \text { on } \partial \Omega^{\delta}, \quad t>0 .
$$

We want to describe the behavior of $\left(E^{\delta}, H^{\delta}\right)$ when $\delta$ tends to 0 . For this, it is useful to apply a change of variables in order to work in a fixed geometry. Doing so, the parameter $\delta$ appears only in the coefficients of the governing equations. We introduce the rescaled fields $\left(\widetilde{E}_{T}^{\delta}, \widetilde{E}_{3}^{\delta}, \widetilde{H}_{T}^{\delta}, \widetilde{H}_{3}^{\delta}\right)$ defined by:

$$
E_{T}^{\delta}=\widetilde{E}_{T}^{\delta} \circ \mathcal{G}_{\delta}^{-1}, \quad E_{3}^{\delta}=\widetilde{E}_{3}^{\delta} \circ \mathcal{G}_{\delta}^{-1}, \quad H_{T}^{\delta}=\widetilde{H}_{T}^{\delta} \circ \mathcal{G}_{\delta}^{-1}, \quad H_{3}^{\delta}=\widetilde{H}_{3}^{\delta} \circ \mathcal{G}_{\delta}^{-1} .
$$

We can write from $(2.11,2.12)$ the equations for $\left(\widetilde{E}_{T}^{\delta}, \widetilde{E}_{3}^{\delta}, \widetilde{H}_{T}^{\delta}, \widetilde{H}_{3}^{\delta}\right)$ in the fixed domain $\Omega$ (the $\delta^{-1}$ terms simply comes from derivatives in $\left(x_{1}, x_{2}\right)$ and $\left.J_{S}=\left(J_{1}, J_{2}\right)^{t}\right)$ :

$$
\begin{cases}\varepsilon \frac{\partial \widetilde{E}_{T}^{\delta}}{\partial t}-\mathbf{e}_{3} \times \frac{\partial \widetilde{H}_{T}^{\delta}}{\partial x_{3}}-\delta^{-1} \overrightarrow{\operatorname{rot}} \widetilde{H}_{3}^{\delta}=J_{S}, & \text { in } \Omega, \quad t>0 \\ \varepsilon \frac{\partial \widetilde{E}_{3}^{\delta}}{\partial t}-\delta^{-1} \operatorname{rot} \widetilde{H}_{T}^{\delta}=0, & \text { in } \Omega, \quad t>0, \\ \mu \frac{\partial \widetilde{H}_{T}^{\delta}}{\partial t}+\mathbf{e}_{3} \times \frac{\partial \widetilde{E}_{T}^{\delta}}{\partial x_{3}}+\delta^{-1} \overrightarrow{\operatorname{rot}} \widetilde{E}_{3}=0, & \text { in } \Omega, t>0, \\ \mu \frac{\partial \widetilde{H}_{3}^{\delta}}{\partial t}+\delta^{-1} \operatorname{rot} \widetilde{E}_{T}^{\delta}=0, & \text { in } \Omega, t>0\end{cases}
$$

while from the divergence equations (2.13),

$$
\begin{cases}\delta^{-1} \operatorname{div} \varepsilon \widetilde{E}_{T}^{\delta}+\varepsilon \frac{\partial \widetilde{E}_{3}^{\delta}}{\partial x_{3}}=0, & \text { in } \Omega, t>0 \\ \delta^{-1} \operatorname{div} \mu \widetilde{H}_{T}^{\delta}+\mu \frac{\partial \widetilde{H}_{3}^{\delta}}{\partial x_{3}}=0, & \text { in } \Omega, t>0 .\end{cases}
$$

The equations (2.16) are completed by zero initial conditions

$$
\widetilde{E}_{T}^{\delta}(\mathbf{x}, 0)=0, \quad \widetilde{E}_{3}^{\delta}(\mathbf{x}, 0)=0, \quad \widetilde{H}_{T}^{\delta}(\mathbf{x}, 0)=0, \quad \widetilde{H}_{3}^{\delta}(\mathbf{x}, 0)=0, \quad \text { a. e. } \mathbf{x} \in \Omega,
$$

and boundary conditions easily deduced from $(2.12,2.14)$

$$
\widetilde{E}_{T}^{\delta} \times n=0, \quad \widetilde{E}_{3}^{\delta}=0, \quad \widetilde{H}_{T}^{\delta} \cdot n=0, \quad \text { on } \partial \Omega, \quad t>0 .
$$

To conclude this section, we recall (without proof) the standard existence, uniqueness and regularity results (see [6] for instance) for the evolution problem $(2.16,2.18,2.19)$ together with a priori estimates that are obtained via standard energy techniques. It is useful to introduce some notation. For integers $(p, q)$, we set

$$
D^{p \cdot q}:=\frac{\partial^{p+q}}{\partial x_{3}^{p} \partial t^{q}}
$$


and for $m$ a strictly positive integer, we introduce the Banach spaces

$$
\begin{aligned}
& \mathcal{W}^{m}(\Omega \times[0, T])=\left\{J \in L^{1}\left(0, T ; L^{2}(\Omega)^{2}\right) / D^{p . q} J \in L^{1}\left(0, T ; L^{2}(\Omega)^{2}\right), p+q \leq m\right\}, \\
& \mathcal{W}_{0}^{m}(\Omega \times[0, T])=\left\{J \in \mathcal{W}^{m}(\Omega \times[0, T]) / D^{0, q} J(\cdot, 0)=0, q \leq m-1\right\} .
\end{aligned}
$$

Theorem 2.1. Assuming that $J_{S}$ satisfies

$$
J_{S} \in \mathcal{W}^{0}(\Omega \times[0, T]):=L^{1}\left([0, T], L^{2}(\Omega)^{2}\right),
$$

the problem $(2.16,2.18,2.19)$ admits a unique solution $\widetilde{E}^{\delta}=\left(\widetilde{E}_{T}^{\delta}, \widetilde{E}_{3}^{\delta}\right), \widetilde{H}^{\delta}=\left(\widetilde{H}_{T}^{\delta}, \widetilde{H}_{3}^{\delta}\right)$ with

$$
\left(\widetilde{E}^{\delta}, \widetilde{H}^{\delta}\right) \in C^{0}\left([0, T], L^{2}(\Omega)^{3}\right)^{2},
$$

which satisfies the a priori estimate (with $C>0$ depending only on $\varepsilon$ and $\mu$ )

$$
\forall t \leq T, \quad\left\|\widetilde{E}^{\delta}(\cdot, t)\right\|_{L^{2}(\Omega)}+\left\|\widetilde{H}^{\delta}(\cdot, t)\right\|_{L^{2}(\Omega)} \leq C \int_{0}^{t}\left\|J_{S}(\cdot, s)\right\|_{L^{2}(\Omega)} d s .
$$

Moreover if $J_{S} \in \mathcal{W}_{0}^{m}(\Omega \times[0, T])$ then,

$$
\left(D^{p \cdot q} \widetilde{E}^{\delta}, D^{p \cdot q} \widetilde{H}^{\delta}\right) \in C^{0}\left([0, T], L^{2}(\Omega)^{3}\right)^{2}, p+q \leq m
$$

and for any $t \leq T$

$$
\left\|D^{p \cdot q} \widetilde{E}^{\delta}(\cdot, t)\right\|_{L^{2}(\Omega)}+\left\|D^{p \cdot q} \widetilde{H}^{\delta}(\cdot, t)\right\|_{L^{2}(\Omega)} \leq C \int_{0}^{t}\left\|D^{p \cdot q} J_{S}(\cdot, s)\right\|_{L^{2}(\Omega)} d s .
$$

\section{Main results}

Before stating the main results of this article, we briefly recap the results from [4] in the particular context of section 2 . To characterize the limit behavior of the electric and magnetic fields $\left(E^{\delta}, H^{\delta}\right)$, we need to introduce $\varphi_{s}(x)$ and $\psi_{s}(x)$ solutions of particular 2D electro-static and magneto-static problems posed in $S$.

More precisely, we define $\varphi_{s} \in H^{1}(S)$ as the unique solution of the problem

$$
\begin{cases}\operatorname{div} \varepsilon \nabla \varphi_{s}=0 & \text { in } S, \\ \varphi_{S}=0 \quad \text { on } \partial S^{+}, & \varphi_{s}=1 \quad \text { on } \partial S^{-} .\end{cases}
$$

and $\psi_{s} \in H^{1}\left(S_{\Gamma}\right)$ as the unique solution of the problem $\left([\cdot]_{\Gamma}\right.$ denoting the jump of a quantity through $\Gamma$ )

$$
\left\{\begin{array}{lr}
\operatorname{div} \mu \nabla \psi_{s}=0 \quad \text { in } S^{\Gamma}, & \int_{S^{\Gamma}} \psi_{s} d x=0, \\
{\left[\mu \nabla \psi_{s} \cdot n\right]_{\Gamma}=0 \quad\left[\psi_{s}\right]_{\Gamma}=1} & \text { on } \Gamma, \nabla \psi_{s} \cdot n=0 \quad \text { on } \partial S .
\end{array}\right.
$$


Remark 3.1. As already emphasized in [4], $\psi_{s}$ depends on the cut $\Gamma$ but not its gradient. More precisely, even though $\psi_{s}$ is not in $H^{1}(S)$, its gradient $\nabla \psi_{s}$ defined in the sense of distribution in $S_{\Gamma}$ defines a vector field in $L^{2}(S)^{2}$ which does not depend on $\Gamma$.

Remark 3.2. In the forthcoming analysis we will used the following property proven in [4]:

$$
\left(\overrightarrow{\operatorname{rot}} \psi_{s}, \nabla \varphi_{s}\right)_{L^{2}(S)}=-\left(\overrightarrow{\operatorname{rot}} \varphi_{s}, \nabla \psi_{s}\right)_{L^{2}(S)}=1
$$

The limit model corresponding to the 3D equations (2.16) will be a $1 D$ wave equation with coefficients, called homogenized coefficients, that are obtained by some kind of "weighted averages" of the original physical coefficients. More precisely we define the capacitance $\mathbf{C}$ and the inductance $\mathbf{L}$ of the cable as coefficients $(\mathbf{C}, \mathbf{L})$ as

$$
\mathbf{C}:=\int_{S} \varepsilon(x)\left|\nabla \varphi_{S}(x)\right|^{2} d x, \quad \mathbf{L}:=\int_{S} \mu(x)\left|\nabla \psi_{S}(x)\right|^{2} d x .
$$

At the (formal) limit $\delta \rightarrow 0$, the electromagnetic field becomes purely transverse:

$$
\begin{aligned}
& E^{\delta}\left(x, x_{3}, t\right) \sim E^{0}\left(x, x_{3}, t\right) \equiv\left(E_{T}^{0}\left(x, x_{3}, t\right), 0\right)^{t} \equiv\left(\widetilde{E}_{T}^{0}\left(x / \delta, x_{3}, t\right), 0\right)^{t}, \quad(\delta \rightarrow 0), \\
& H^{\delta}\left(x, x_{3}, t\right) \sim H^{0}\left(x, x_{3}, t\right) \equiv\left(H_{T}^{0}\left(x, x_{3}, t\right), 0\right)^{t} \equiv\left(\widetilde{H}_{T}^{0}\left(x / \delta, x_{3}, t\right), 0\right)^{t}, \quad(\delta \rightarrow 0) .
\end{aligned}
$$

where the limit transverse electric field $\widetilde{E}_{T}^{0}$ and the limit transverse magnetic field $\widetilde{H}_{T}^{0}$ are given by (this means in particular that one has asymptotically separation of variables between $x$ and $x_{3}$ )

$$
\left\{\begin{array}{l}
\widetilde{E}_{T}^{0}\left(x, x_{3}, t\right)=V\left(x_{3}, t\right) \nabla \varphi_{s}(x), \\
\widetilde{H}_{T}^{0}\left(x, x_{3}, t\right)=I\left(x_{3}, t\right) \nabla \psi_{s}(x),
\end{array}\right.
$$

where $V\left(x_{3}, t\right)$ and $I\left(x_{3}, t\right)$ are the solutions of

$$
\begin{cases}\mathbf{C} \frac{\partial V}{\partial t}\left(x_{3}, t\right)+\frac{\partial I}{\partial x_{3}}\left(x_{3}, t\right)=I_{S}\left(x_{3}, t\right), & \text { in } \mathbb{R}, t>0 \\ \mathbf{L} \frac{\partial I}{\partial t}\left(x_{3}, t\right)+\frac{\partial V}{\partial x_{3}}\left(x_{3}, t\right)=0, & \text { in } \mathbb{R}, t>0\end{cases}
$$

with $(\mathbf{C}, \mathbf{L})$ defined by (3.4) and where $I_{S}$ is defined by

$$
I_{S}\left(x_{3}, t\right)=\int_{S} J_{S}(x, t) \cdot \nabla \varphi_{S}(x) d x
$$

The equations (3.7) are naturally completed with zero initial conditions

$$
V\left(x_{3}, 0\right)=I\left(x_{3}, 0\right)=0, \quad x_{3} \in \mathbb{R} .
$$

We now state for the limit 1D problem, the equivalent of theorem (2.1) (existence, uniqueness, regularity). 
Theorem 3.1. Assuming that $J_{S}$ satisfies

$$
J_{S} \in \mathcal{W}^{0}(\Omega \times[0, T]),
$$

then the problem $(3.7,3.8,3.9)$ admits a unique solution

$$
V \in C^{0}\left(0, T ; L^{2}(\mathbb{R})\right), \quad I \in C^{0}\left(0, T ; L^{2}(\mathbb{R})\right) .
$$

which satisfies the a priori estimate $(C>0$ depends only on $(S, \varepsilon, \mu))$

$$
\|V(\cdot, t)\|_{L^{2}(\mathbb{R})}+\|I(\cdot, t)\|_{L^{2}(\mathbb{R})} \leq C \int_{0}^{t}\left\|J_{S}(\cdot, s)\right\|_{L^{2}(\Omega)} d s,
$$

If, moreover, $J_{S}$ satisfies

$$
J_{S} \in \mathcal{W}^{1}(\Omega \times[0, T]),
$$

then $(V, I) \in\left(C^{1}\left(0, T: L^{2}(\mathbb{R})\right) \cap C^{0}\left(0, T: L^{2}(\mathbb{R})\right)^{2}\right.$ and for any $t \leq T$ :

$$
\begin{gathered}
\left\|\frac{\partial V}{\partial t}(\cdot, t)\right\|_{L^{2}(\mathbb{R})}+\left\|\frac{\partial I}{\partial t}(\cdot, t)\right\|_{L^{2}(\mathbb{R})} \leq C \int_{0}^{t}\left\|\frac{\partial J_{S}}{\partial t}(\cdot, s)\right\|_{L^{2}(\Omega)} d s \\
\left\|\frac{\partial V}{\partial x_{3}}(\cdot, t)\right\|_{L^{2}(\mathbb{R})}+\left\|\frac{\partial J_{S}}{\partial x_{3}}(\cdot, t)\right\|_{L^{2}(\mathbb{R})} \leq C \int_{0}^{t}\left\|\frac{\partial J_{S}}{\partial x_{3}}(\cdot, s)\right\|_{L^{2}(\Omega)} d s .
\end{gathered}
$$

We set

$$
\left|J_{S}\right|_{1,1, T}:=\left\|\frac{\partial J_{S}}{\partial t}(\cdot, s)\right\|_{L^{1}\left(0, T ; L^{2}(\Omega)\right)}+\left\|\frac{\partial J_{S}}{\partial x_{3}}(\cdot, s)\right\|_{L^{1}\left(0, T ; L^{2}(\Omega)\right)^{\prime}},
$$

and more generally, for arbitrary integer $p \geq 1$ and $q \geq 1$

$$
\left|J_{S}\right|_{p, q, T}:=\left\|D^{p-1 . q} J_{S}\right\|_{L^{1}\left(0, T ; L^{2}(\Omega)\right)}+\left\|D^{p \cdot q-1} J_{S}\right\|_{L^{1}\left(0, T ; L^{2}(\Omega)\right)} \cdot
$$

We are now in position to give the main results of this article.

Theorem 3.2. Assuming that $J_{S} \in \mathcal{W}_{0}^{1}(\Omega \times[0, T])$ (cf. 2.20), then

$$
\left\|\widetilde{E}_{3}^{\delta}\right\|_{L^{\infty}\left(0, T ; H^{1}(\Omega)\right)}+\left\|\widetilde{H}_{3}^{\delta}\right\|_{L^{\infty}\left(0, T ; H^{1}(\Omega)\right)} \leq C \delta\left|J_{s}\right|_{1,1, T}
$$

and for the transverse fields

$$
\left\|\widetilde{E}_{T}^{\delta}-\widetilde{E}_{T}^{0}\right\|_{L^{\infty}\left(0, T ; L^{2}(\Omega)\right)}+\left\|\widetilde{H}_{T}^{\delta}-\widetilde{H}_{T}^{0}\right\|_{L^{\infty}\left(0, T ; L^{2}(\Omega)\right)} \leq C \delta^{\frac{1}{2}}\left(\delta^{\frac{1}{2}}+T^{\frac{1}{2}}\right)\left|J_{s}\right|_{1,1, T} .
$$

If, in addition, $J_{S} \in \mathcal{W}_{0}^{2}(\Omega \times[0, T])$ then

$$
\left\|\widetilde{E}_{T}^{\delta}-\widetilde{E}_{T}^{0}\right\|_{L^{\infty}\left(0, T ; L^{2}(\Omega)\right)}+\left\|\widetilde{H}_{T}^{\delta}-\widetilde{H}_{T}^{0}\right\|_{L^{\infty}\left(0, T ; L^{2}(\Omega)\right)} \leq C \delta\left(\left|J_{s}\right|_{1,1, T}+T\left|J_{s}\right|_{2,1, T}\right) .
$$

Finally, if $J_{S} \in \mathcal{W}_{0}^{3}(\Omega \times[0, T])$ then

$$
\begin{aligned}
& \left\|\widetilde{E}_{T}^{\delta}-\widetilde{E}_{T}^{0}\right\|_{L^{\infty}\left(0, T ; L^{2}(\Omega)\right)}+\left\|\widetilde{H}_{T}^{\delta}-\widetilde{H}_{T}^{0}\right\|_{L^{\infty}\left(0, T ; L^{2}(\Omega)\right)} \\
& \quad \leq C \delta^{2}\left(\left|J_{s}\right|_{1,2, T}+\left|J_{s}\right|_{2,1, T}+T\left(\left|J_{s}\right|_{2,2, T}+\left|J_{s}\right|_{3,1, T}\right)\right) .
\end{aligned}
$$




\section{Proof of the main theorem}

We decompose the proof in several steps. As usual, in what follows, $C$ will denote a generic positive scalar that may change from one line to the other. Unless specifically mentioned, $C$ depends only on $(S, \varepsilon, \mu)$. Note that, with the regularity assumption on the source term $J_{S}$, we deduce from theorem 2.1 and the equations (2.5) that the solution of the full 3D problem has the regularity:

$$
\left(\widetilde{E}^{\delta}, \widetilde{H}^{\delta}\right) \in C^{1}\left(0, T ; L^{2}(\Omega)^{3}\right)^{2}, \quad\left(\nabla \times \widetilde{E}^{\delta}, \nabla \times \widetilde{H}^{\delta}\right) \in C^{0}\left(0, T ; L^{2}(\Omega)^{3}\right)^{2} .
$$

Step 1 : Proof of the estimate (3.13) for the longitudinal fields.

This step is quite immediate. Using the first and the third equations of (2.16), we immediately get

$$
\begin{gathered}
\left\|\nabla \widetilde{H}_{3}^{\delta}(\cdot, t)\right\|_{L^{2}(\Omega)} \leq C \delta\left(\left\|\frac{\partial \widetilde{E}_{T}^{\delta}}{\partial t}(\cdot, t)\right\|_{L^{2}(\Omega)}+\left\|\frac{\partial \widetilde{H}_{T}^{\delta}}{\partial x_{3}}(\cdot, t)\right\|_{L^{2}(\Omega)}\right), \\
\left\|\nabla \widetilde{E}_{3}^{\delta}(\cdot, t)\right\|_{L^{2}(\Omega)} \leq C \delta\left(\left\|\frac{\partial \widetilde{H}_{T}^{\delta}}{\partial t}(\cdot, t)\right\|_{L^{2}(\Omega)}+\left\|\frac{\partial \widetilde{E}_{T}^{\delta}}{\partial x_{3}}(\cdot, t)\right\|_{L^{2}(\Omega)}\right),
\end{gathered}
$$

so that, using the stability estimates (2.22) of theorem 2.1

$$
\left\|\nabla \widetilde{H}_{3}^{\delta}\right\|_{L^{\infty}\left(0, T ; L^{2}(\Omega)\right)}+\left\|\nabla \widetilde{E}_{3}^{\delta}\right\|_{L^{\infty}\left(0, T ; L^{2}(\Omega)\right)} \leq C \delta\left|J_{s}\right|_{1,1, T}
$$

To conclude, it suffices to use Poincaré's type inequalities.

For the electric field, (3.13) results from the classical Poincaré's inequality since by (2.19) (second equation) $E_{3}^{\delta}\left(\cdot, x_{3}, t\right)$ belongs to $H_{0}^{1}(S)$ for each $t>0$ and almost every $x_{3} \in \mathbb{R}$. For the magnetic field, we can use a (generalized) Poincaré-Wirtinger's inequality since :

$$
\int_{S} \mu(x) \widetilde{H}_{3}^{\delta}\left(x, x_{3}, t\right) d x=0, \quad \forall 0 \leq t \leq T, \quad \text { a. e. } x_{3} \in \mathbb{R} .
$$

This is obtained by integrating over $\Omega$ the fourth equation of (2.16), after multiplication by a smooth $1 \mathrm{D}$ test function with compact support $\varphi\left(x_{3}\right)$. This gives, using Green's formula,

$$
\begin{aligned}
\frac{d}{d t}\left(\int_{\Omega} \mu \widetilde{H}_{3}^{\delta} \varphi d \mathbf{x}\right) & =-\delta^{-1} \int_{\mathbb{R}}\left(\int_{S} \operatorname{rot} \widetilde{E}_{T}^{\delta}\left(x, x_{3}, t\right) d x\right) \varphi\left(x_{3}\right) d x_{3} \\
& =\delta^{-1} \int_{\mathbb{R}}\left\langle E_{T}^{\delta} \times n, \varphi\left(x_{3}\right)\right\rangle_{S} d x_{3}=0,
\end{aligned}
$$

thanks to the boundary condition (2.19) (first equation). (4.2) follows easily since $H_{3}^{\delta}$ vanishes at time $t=0$. 
It is then easy to obtained analogous estimates for derivatives in $x_{3}$ and $t$ of these longitudinal fields. Indeed, since all coefficients in equations (2.16) are independent of $x_{3}$ and $t$, it is clear that the fields $D^{p, q} \widetilde{E}^{\delta}$ and $D^{p, q} \widetilde{H}^{\delta}$ are related to $D^{p, q} \widetilde{J}_{S}$ by the same partial differential equations that the ones which link $\widetilde{E}^{\delta}$ and $\widetilde{H}^{\delta}$ to $\widetilde{J}_{S}$. Moreover, since the domain $\Omega$ is a cylinder, they satisfy the same homogeneous boundary condition. Finally, provided that time derivatives of $J_{S}$ or order less or equal to $q-1$ vanish, these fields vanish at time $t=0$. From this remarks, the reader will easily check that, if

$$
J_{S} \in \mathcal{W}_{0}^{m}(\Omega \times[0, T]),
$$

then for any $p+q \leq m$

$$
\left\|D^{p, q} \widetilde{E}_{3}^{\delta}\right\|_{L^{\infty}\left(0, T ; H^{1}(\Omega)\right)}+\left\|D^{p, q} \widetilde{H}_{3}^{\delta}\right\|_{L^{\infty}\left(0, T ; H^{1}(\Omega)\right)} \leq C \delta\left|D^{p, q} J_{s}\right|_{1,1, T} .
$$

Step 2 : decomposition of the transverse fields and related Poincaré-Friedrichs inequalities.

The decomposition we shall use is related to the following orthogonal decomposition of spaces of square integrable $2 \mathrm{D}$ vector fields in S. Concerning the transverse electric field, we first define the Hilbert spaces:

$$
\begin{aligned}
& V(\varepsilon) \equiv L^{2}(S)^{2} \text { equipped with the inner product }(u, v)_{\varepsilon}:=\int_{S} \varepsilon u \cdot v d x, \\
& W(\varepsilon)=\left\{u \in L^{2}(S)^{2} / \operatorname{div} \varepsilon u \in L^{2}(S), \operatorname{rot} u \in L^{2}(S), u \times n=0 \text { on } \partial S\right\} .
\end{aligned}
$$

$V(\varepsilon)$ can be decomposed as (the decomposition is orthogonal with respect to $(u, v)_{\varepsilon}$ )

$$
V(\varepsilon)=U(\varepsilon) \oplus U(\varepsilon)^{\perp}, \quad \text { where } U(\varepsilon):=\operatorname{span}\left[\nabla \varphi_{s}\right] .
$$

In [4], it has been shown that $U(\varepsilon)$ is characterized by

$$
U(\varepsilon)=\{u \in W(\varepsilon) / \operatorname{div} \varepsilon u=0, \operatorname{rot} u=0\},
$$

a result which is related to the following Poincaré-Friedrichs inequality (see the appendix for the proof), is:

Proposition 4.1. There exists $C>0$ depending only on $(S, \varepsilon)$ such that,

$$
\forall u \in W(\varepsilon), \quad\|u\|_{L^{2}(S)} \leq C\left(\|\operatorname{rot} u\|_{L^{2}(S)}+\|\operatorname{div} \varepsilon u\|_{L^{2}(S)}+\left|\left(u, \nabla \varphi_{S}\right)_{\varepsilon}\right|\right) .
$$

In the same way, for the transverse magnetic field, we first define the Hilbert spaces:

$$
\begin{aligned}
& V(\mu) \equiv L^{2}(S)^{2} \text { equipped with the inner product }(u, v)_{\mu}:=\int_{S} \mu u \cdot v d x, \\
& W(\mu)=\left\{u \in L^{2}(S)^{2} / \operatorname{div} \mu u \in L^{2}(S), \operatorname{rot} u \in L^{2}(S), u \cdot n=0 \text { on } \partial S\right\} .
\end{aligned}
$$

$V(\mu)$ can be decomposed as (the decomposition is orthogonal with respect to $\left.(u, v)_{\mu}\right)$

$$
V(\mu)=U(\mu) \oplus U(\mu)^{\perp}, \quad \text { where } U(\mu):=\operatorname{span}\left[\nabla \psi_{s}\right] .
$$


In [4], it has been shown that $U(\mu)$ is characterized by

$$
U(\mu)=\{u \in W(\mu) / \operatorname{div} \mu u=0, \operatorname{rot} u=0\} .
$$

It is possible to show the following Poincaré-Friedrichs inequality (that we assumed here but can be rigorously proven)

Proposition 4.2. There exist $C>0$ depending only on $(S, \mu)$ such that,

$$
\forall u \in W(\mu), \quad\|u\|_{L^{2}(S)} \leq C\left(\|\operatorname{rot} u\|_{L^{2}(S)}+\|\operatorname{div} \mu u\|_{L^{2}(S)}+\left|\left(u, \nabla \psi_{S}\right)_{\mu}\right|\right) .
$$

According to the orthogonal decompositions (4.5) and (4.9), for each $\left(x_{3}, t\right)$, the transverse fields $\widetilde{E}_{T}^{\delta}\left(\cdot, x_{3}, t\right)$ and $\widetilde{H}_{T}^{\delta}\left(\cdot, x_{3}, t\right)$ will be splitted as follows :

$$
\begin{array}{ll}
\widetilde{E}_{T}^{\delta}\left(\cdot, x_{3}, t\right)=V^{\delta}\left(x_{3}, t\right) \nabla \varphi_{s}+\widetilde{E}_{T}^{R, \delta}\left(\cdot, x_{3}, t\right), & \widetilde{E}_{T}^{R, \delta}\left(\cdot, x_{3}, t\right) \in U(\varepsilon)^{\perp}, \\
\widetilde{H}_{T}^{\delta}\left(\cdot, x_{3}, t\right)=I^{\delta}\left(x_{3}, t\right) \nabla \psi_{s}+\widetilde{H}_{T}^{R, \delta}\left(\cdot, x_{3}, t\right), & \widetilde{H}_{T}^{R, \delta}\left(\cdot, x_{3}, t\right) \in U(\mu)^{\perp} .
\end{array}
$$

where, from the orthogonality of the decompositions, the definitions (3.4) and equalities (4.11), the scalar quantities $I^{\delta}\left(x_{3}, t\right)$ and $V^{\delta}\left(x_{3}, t\right)$ are given by

$$
\begin{aligned}
& V^{\delta}\left(x_{3}, t\right)=\mathbf{C}^{-1}\left(\widetilde{E}_{T}^{\delta}\left(\cdot, x_{3}, t\right), \nabla \varphi_{s}\right)_{\varepsilon}, \\
& I^{\delta}\left(x_{3}, t\right)=\mathbf{L}^{-1}\left(\widetilde{H}_{T}^{\delta}\left(\cdot, x_{3}, t\right), \nabla \psi_{s}\right)_{\mu} .
\end{aligned}
$$

According to $(3.5,3.6)$, we expect that the "residual" transverse fields $\widetilde{E}_{T}^{R, \delta}$ and $\widetilde{H}_{T}^{R, \delta}$ converge to 0 when $\delta \rightarrow 0$ while $V^{\delta}\left(x_{3}, t\right)$ and $I^{\delta}\left(x_{3}, t\right)$ converge to $V\left(x_{3}, t\right)$ and $I\left(x_{3}, t\right)$ (the solutions of $(3.7,3.8,3.9))$. This is exactly the way the error estimates (3.14) and (3.15) will be proven in the next two steps, using the triangular inequality:

$$
\begin{aligned}
& \left\|E_{T}^{\delta}-\widetilde{E}_{T}^{0}\right\|_{L^{\infty}\left(0, T ; L^{2}(\Omega)\right)} \leq\left\|\widetilde{E}_{T}^{R, \delta}\right\|_{L^{\infty}\left(0, T ; L^{2}(\Omega)\right)}+\left\|\left(V-V^{\delta}\right) \nabla \varphi_{s}\right\|_{L^{\infty}\left(0, T ; L^{2}(\Omega)\right)}, \\
& \left\|H_{T}^{\delta}-\widetilde{H}_{T}^{0}\right\|_{L^{\infty}\left(0, T ; L^{2}(\Omega)\right)} \leq\left\|\widetilde{H}_{T}^{R, \delta}\right\|_{L^{\infty}\left(0, T ; L^{2}(\Omega)\right)}+\left\|\left(I-I^{\delta}\right) \nabla \psi_{s}\right\|_{L^{\infty}\left(0, T ; L^{2}(\Omega)\right)},
\end{aligned}
$$

which yields by a straightforward calculation exploiting the separation of variables:

$$
\begin{aligned}
& \left\|E_{T}^{\delta}-\widetilde{E}_{T}^{0}\right\|_{L^{\infty}\left(0, T ; L^{2}(\Omega)\right)} \leq\left\|\widetilde{E}_{T}^{R, \delta}\right\|_{L^{\infty}\left(0, T ; L^{2}(\Omega)\right)}+C\left\|\left(V-V^{\delta}\right)\right\|_{L^{\infty}\left(0, T ; L^{2}(\mathbb{R})\right)}, \\
& \left\|H_{T}^{\delta}-\widetilde{H}_{T}^{0}\right\|_{L^{\infty}\left(0, T ; L^{2}(\Omega)\right)} \leq\left\|\widetilde{H}_{T}^{R, \delta}\right\|_{L^{\infty}\left(0, T ; L^{2}(\Omega)\right)}+C\left\|\left(I-I^{\delta}\right)\right\|_{L^{\infty}\left(0, T ; L^{2}(\mathbb{R})\right)} .
\end{aligned}
$$

Step 3 : estimates of the residual transverse fields.

First note that, from the boundary conditions $(2.12,2.14)$ and the definitions of $\left(\varphi_{s}, \psi_{s}\right)$ we deduce the boundary equations

$$
E_{T}^{R, \delta} \times n=0, \quad \widetilde{H}_{T}^{R, \delta} \cdot n=0 .
$$


Thus as consequences of Poincaré-Friedriches inequalities (4.7) and (4.10), we deduce that

$$
\begin{gathered}
\left\|\widetilde{E}_{T}^{R, \delta}\right\|_{L^{2}(\Omega)} \leq C\left(\left\|\operatorname{rot} \widetilde{E}_{T}^{R, \delta}\right\|_{L^{2}(\Omega)}+\left\|\operatorname{div} \varepsilon \widetilde{E}_{T}^{R, \delta}\right\|_{L^{2}(\Omega)}\right), \\
\left\|\widetilde{H}_{T}^{R, \delta}\right\|_{L^{2}(\Omega)} \leq C\left(\left\|\operatorname{rot} \widetilde{H}_{T}^{R, \delta}\right\|_{L^{2}(\Omega)}+\left\|\operatorname{div} \mu \widetilde{H}_{T}^{R, \delta}\right\|_{L^{2}(\Omega)}\right) .
\end{gathered}
$$

Moreover, from the definitions of $\widetilde{E}_{T}^{R, \delta}$ and $\widetilde{H}_{T}^{R, \delta}$ given by (4.11), as well as from the definition of $\widehat{\varphi}_{s}$ and $\widehat{\psi}_{s}$ given by $(3.1,3.2)$ we have

$$
\begin{aligned}
& \operatorname{rot} \widetilde{E}_{T}^{R, \delta}=\operatorname{rot} \widetilde{E}_{T}^{\delta}, \quad \operatorname{div} \varepsilon \widetilde{E}_{T}^{R, \delta}=\operatorname{div} \varepsilon \widetilde{E}_{T}^{\delta}, \\
& \operatorname{rot} \widetilde{H}_{T}^{R, \delta}=\operatorname{rot} \widetilde{H}_{T}^{\delta}, \quad \operatorname{div} \mu \widetilde{H}_{T}^{R, \delta}=\operatorname{div} \mu \widetilde{H}_{T}^{\delta} .
\end{aligned}
$$

Using the second and the fourth equations of (2.16), this implies that for any $t \leq T$,

$$
\begin{aligned}
& \left\|\operatorname{rot} \widetilde{H}_{T}^{R, \delta}(\cdot, t)\right\|_{L^{2}(\Omega)}=\delta\left\|\varepsilon \frac{\partial}{\partial t} \widetilde{E}_{3}^{\delta}(\cdot, t)\right\|_{L^{2}(\Omega)} \leq C \delta \int_{0}^{t}\left\|\frac{\partial J_{S}}{\partial t}(\cdot, s)\right\|_{L^{2}(\Omega)} d s, \\
& \left\|\operatorname{rot} \widetilde{E}_{T}^{R, \delta}(\cdot, t)\right\|_{L^{2}(\Omega)}=\delta\left\|\mu \frac{\partial}{\partial t} \widetilde{H}_{3}^{\delta}(\cdot, t)\right\|_{L^{2}(\Omega)} \leq C \delta \int_{0}^{t}\left\|\frac{\partial J_{S}}{\partial t}(\cdot, s)\right\|_{L^{2}(\Omega)} d s .
\end{aligned}
$$

Moreover, using the "hidden" equation (2.17):

$$
\begin{aligned}
& \left\|\operatorname{div} \varepsilon \widetilde{E}_{T}^{R, \delta}\right\|_{L^{2}(\Omega)}=\delta\left\|\varepsilon \frac{\partial \widetilde{E}_{3}^{\delta}}{\partial x_{3}}\right\|_{L^{2}(\Omega)} \leq C \delta \int_{0}^{t}\left\|\frac{\partial J_{S}}{\partial x_{3}}(\cdot, s)\right\|_{L^{2}(\Omega)} d s, \\
& \left\|\operatorname{div} \mu \widetilde{H}_{T}^{R, \delta}\right\|_{L^{2}(\Omega)}=\delta\left\|\mu \frac{\partial \widetilde{H}_{3}^{\delta}}{\partial x_{3}}\right\|_{L^{2}(\Omega)} \leq C \delta \int_{0}^{t}\left\|\frac{\partial J_{S}}{\partial x_{3}}(\cdot, s)\right\|_{L^{2}(\Omega)} d s .
\end{aligned}
$$

Substituting (4.17) and (4.18) into (4.15), we easily deduce that

$$
\left\|\widetilde{E}_{T}^{R, \delta}\right\|_{L^{\infty}\left(0, T ; L^{2}(\Omega)\right)}+\left\|\widetilde{H}_{T}^{R, \delta}\right\|_{L^{\infty}\left(0, T ; L^{2}(\Omega)\right)} \leq C \delta\left|J_{S}\right|_{1,1, T} .
$$

Moreover, with one more degree of regularity on the source, namely if one assumes that $J_{S} \in \mathcal{W}_{0}^{2}(\Omega \times[0, T])$, such estimate is easily extended into analogous estimate about the $x_{3}$-derivatives of the residual transverse fields (the details are left to the reader):

$$
\left\|\frac{\partial \widetilde{E}_{T}^{R, \delta}}{\partial x_{3}}\right\|_{L^{\infty}\left(0, T ; L^{2}(\Omega)\right)}+\left\|\frac{\partial \widetilde{H}_{T}^{R, \delta}}{\partial x_{3}}\right\|_{L^{\infty}\left(0, T ; L^{2}(\Omega)\right)} \leq C \delta\left|J_{S}\right|_{2,1, T}
$$

We can improve these estimates. Indeed, if $J_{S} \in \mathcal{W}_{0}^{2}(\Omega \times[0, T])$, we can apply the estimate (4.3) with $(p, q)=(1,0)$ and $(p, q)=(1,0)$ to obtain $O(\delta)$ upper bounds for first order derivatives in $x_{3}$ and $t$ of $\widetilde{E}_{3}^{\delta}$ and $\widetilde{H}_{3}^{\delta}$. Then, the reader will easily verify that substituting these inequalities into (4.17) and (4.18) and finally into (4.15) leads to

$$
\left\|\widetilde{E}_{T}^{R, \delta}\right\|_{L^{\infty}\left(0, T ; L^{2}(\Omega)\right)}+\left\|\widetilde{H}_{T}^{R, \delta}\right\|_{L^{\infty}\left(0, T ; L^{2}(\Omega)\right)} \leq C \delta^{2}\left(\left|J_{S}\right|_{2,1, T}+\left|J_{S}\right|_{1,2, T}\right) .
$$


Finally, with one more degree of regularity on the source, namely $J_{S} \in \mathcal{W}_{0}^{3}(\Omega \times[0, T])$, this estimate can extended, as before, into estimate about the $x_{3}$-derivatives of the residual transverse fields:

$$
\left\|\frac{\partial \widetilde{E}_{T}^{R, \delta}}{\partial x_{3}}\right\|_{L^{\infty}\left(0, T ; L^{2}(\Omega)\right)}+\left\|\frac{\partial \widetilde{H}_{T}^{R, \delta}}{\partial x_{3}}\right\|_{L^{\infty}\left(0, T ; L^{2}(\Omega)\right)} \leq C \delta^{2}\left(\left|J_{S}\right|_{3,1, T}+\left|J_{S}\right|_{2,2, T}\right) .
$$

Step 4 : proof of the error estimate (3.14).

We first write an equation in $\left(v^{\delta}, i^{\delta}\right)$ defined by

$$
v^{\delta}=V^{\delta}-V \text { and } i^{\delta}=I^{\delta}-I \text {. }
$$

Multiplying the first equation of (2.16) by $\nabla \varphi_{s}$ and the third one by $\nabla \psi_{s}$ and integrating over the section $S$, we obtain (the details are left to the reader)

$$
\left\{\begin{array}{cc}
\mathbf{C} \frac{\partial V^{\delta}}{\partial t}+\left(\overrightarrow{\operatorname{rot}} \psi_{s}, \nabla \varphi_{s}\right)_{L^{2}(S)} \frac{\partial I^{\delta}}{\partial x_{3}} & \text { in } \mathbb{R}, t>0, \\
-\delta^{-1}\left(\overrightarrow{\operatorname{rot}} \widetilde{H}_{3}^{\delta}, \nabla \varphi_{s}\right)_{L^{2}(S)}=\left(\frac{\partial \widetilde{H}_{T}^{R, \delta}}{\partial x_{3}}, \overrightarrow{\operatorname{rot}} \varphi_{s}\right)_{L^{2}(S)}+I_{S}, & \\
\mathbf{L} \frac{\partial I^{\delta}}{\partial t}-\left(\overrightarrow{\operatorname{rot}} \varphi_{s}, \nabla \psi_{s}\right)_{L^{2}(S)} \frac{\partial V^{\delta}}{\partial x_{3}} & \text { in } \mathbb{R}, t>0 \\
+\delta^{-1}\left(\overrightarrow{\operatorname{rot}} \widetilde{E}_{3}^{\delta}, \nabla \psi_{s}\right)_{L^{2}(S)}=-\left(\frac{\partial \widetilde{E}_{T}^{R, \delta}}{\partial x_{3}}, \overrightarrow{\operatorname{rot}} \psi_{s}\right)_{L^{2}(S)} . &
\end{array}\right.
$$

Some important simplifications now occur. First, one can use Green's formula (2.10) and the fact that $\nabla \varphi_{s} \times n=0$ along $\partial \Omega$, to obtain

$$
\left(\overrightarrow{\operatorname{rot}} \widetilde{H}_{3}^{\delta}, \nabla \varphi_{s}\right)_{L^{2}(S)}=\left(\widetilde{H}_{3}^{\delta}, \operatorname{rot} \nabla \varphi_{s}\right)_{L^{2}(S)}+\left\langle\nabla \varphi_{s} \times n, \widetilde{H}_{3}^{\delta}\right\rangle_{\partial S}=0
$$

Next, again using Green's formula (2.10), where $S$ is replaced by $S_{\Gamma}$, as well as the boundary condition (2.19), which gives $\widetilde{E}_{3}^{\delta}=0$ along $\partial \Omega$ we get, with an appropriate orientation of the normal vector $n$ along $\Gamma$,

$$
\begin{aligned}
& \left(\overrightarrow{\operatorname{rot}} \widetilde{E}_{3}^{\delta}, \nabla \psi_{s}\right)_{L^{2}(S)} \equiv\left(\overrightarrow{\operatorname{rot}} \widetilde{E}_{3}^{\delta}, \nabla \psi_{s}\right)_{L^{2}\left(S_{\Gamma}\right)} \\
& \quad=\left(\widetilde{E}_{3}^{\delta}, \operatorname{rot} \nabla \psi_{s}\right)_{L^{2}\left(S_{\Gamma}\right)}+\left\langle\left[\nabla \psi_{s} \times n\right]_{\Gamma}, \widetilde{E}_{3}^{\delta}\right\rangle_{\Gamma}+\left\langle\nabla \psi_{s} \times n, E_{3}^{\delta}\right\rangle_{\partial S}=0 .
\end{aligned}
$$

which implies

$$
\left(\overrightarrow{\operatorname{rot}} E_{3}^{\delta}, \nabla \psi_{s}\right)_{L^{2}(S)}=0,
$$

since $\left[\psi_{s}\right]_{\Gamma}=1$ induces $\left[\nabla \psi_{s} \times n\right]_{\Gamma}=0$. Finally, we use (3.3), to end up with

$$
\begin{cases}\mathbf{C} \frac{\partial V^{\delta}}{\partial t}+\frac{\partial I^{\delta}}{\partial x_{3}}=\left(\frac{\partial \widetilde{H}_{T}^{R, \delta}}{\partial x_{3}}, \overrightarrow{\operatorname{rot}} \varphi_{S}\right)_{L^{2}(S)}+I_{S}, & \text { in } \mathbb{R}, t>0 \\ \mathbf{L} \frac{\partial I^{\delta}}{\partial t}+\frac{\partial V^{\delta}}{\partial x_{3}}=-\left(\frac{\partial \widetilde{E}_{T}^{R, \delta}}{\partial x_{3}}, \overrightarrow{\operatorname{rot}} \psi_{s}\right)_{L^{2}(S)^{\prime}} \quad \text { in } \mathbb{R}, t>0\end{cases}
$$


Subtracting (3.7) to (4.25), we find that $\left(v^{\delta}, i^{\delta}\right)$ are solution of

$$
\begin{cases}\mathbf{C} \frac{\partial v^{\delta}}{\partial t}+\frac{\partial i^{\delta}}{\partial x_{3}}=\left(\frac{\partial \widetilde{H}_{T}^{R, \delta}}{\partial x_{3}}, \overrightarrow{\operatorname{rot}} \varphi_{s}\right)_{L^{2}(S)^{\prime}} & \text { in } \mathbb{R}, \quad t>0 \\ \mathbf{L} \frac{\partial i^{\delta}}{\partial t}+\frac{\partial v^{\delta}}{\partial x_{3}}=-\left(\frac{\partial \widetilde{E}_{T}^{R, \delta}}{\partial x_{3}}, \overrightarrow{\operatorname{rot}} \psi_{s}\right)_{L^{2}(S)^{\prime}} & \text { in } \mathbb{R}, \quad t>0\end{cases}
$$

To prove the convergence result of theorem 3.2, we will proceed using energy techniques. We define the energy

$$
\mathcal{E}^{\delta}(t)=\mathbf{C} \int_{\mathbb{R}} v^{\delta}\left(x_{3}, t\right)^{2} d x_{3}+\mathbf{L} \int_{\mathbb{R}} i^{\delta}\left(x_{3}, t\right)^{2} d x_{3},
$$

where, thanks to the zero initial data, we have $\mathcal{E}^{\delta}(0)=0$. Next, we apply standard energy analysis: we multiply the two equations of (4.26) respectively by $v^{\delta}$ and $i^{\delta}$, we integrate along the $x_{3}$ axis. After integration by parts (in the left hand sides) and summation, we obtain

$$
\frac{1}{2} \frac{d}{d t} \mathcal{E}^{\delta}=\int_{\mathbb{R}}\left(\frac{\partial \widetilde{H}_{T}^{R, \delta}}{\partial x_{3}}, i^{\delta} \overrightarrow{\operatorname{rot}} \varphi_{s}\right)_{L^{2}(S)} d x_{3}-\int_{\mathbb{R}}\left(\frac{\partial \widetilde{E}_{T}^{R, \delta}}{\partial x_{3}}, v^{\delta} \overrightarrow{\operatorname{rot}} \psi_{s}\right)_{L^{2}(S)} d x_{3}
$$

Then, we integrate by parts the right hand side to get

$$
\frac{1}{2} \frac{d}{d t} \mathcal{E}^{\delta}=\int_{\mathbb{R}}\left(\widetilde{E}_{T}^{R, \delta}, \frac{\partial i^{\delta}}{\partial x_{3}} \overrightarrow{\operatorname{rot}} \psi_{s}\right)_{L^{2}(S)} d x_{3}-\int_{\mathbb{R}}\left(\widetilde{H}_{T}^{R, \delta}, \frac{\partial v^{\delta}}{\partial x_{3}} \overrightarrow{\operatorname{rot}} \varphi_{s}\right)_{L^{2}(S)} d x_{3} .
$$

Using Cauchy-Schwarz inequality, we obtain

$$
\frac{1}{2} \frac{d}{d t} \mathcal{E}^{\delta} \leq\left\|\frac{\partial v^{\delta}}{\partial x_{3}} \nabla \varphi_{s}^{\infty}\right\|_{L^{2}(\Omega)}\left\|E_{T}^{R, \delta}\right\|_{L^{2}(\Omega)}+\left\|\frac{\partial i^{\delta}}{\partial x_{3}} \nabla \psi_{s}^{\infty}\right\|_{L^{2}(\Omega)}\left\|\widetilde{H}_{T}^{R, \delta}\right\|_{L^{2}(\Omega)} .
$$

On one hand, thanks to the orthogonal decomposition (4.11), we have

$$
\left\|\frac{\partial \widetilde{E}_{T}^{\delta}}{\partial x_{3}}(\cdot, t)\right\|_{\varepsilon}^{2}=\left\|\frac{\partial V^{\delta}}{\partial x_{3}}(\cdot, t) \nabla \varphi_{s}^{\infty}\right\|_{\varepsilon}^{2}+\left\|\frac{\partial \widetilde{E}_{T}^{R, \delta}}{\partial x_{3}}(\cdot, t)\right\|_{\varepsilon}^{2},
$$

which yields

$$
\left\|\frac{\partial V^{\delta}}{\partial x_{3}}(\cdot, t) \nabla \varphi_{s}^{\infty}\right\|_{L^{2}(\Omega)} \leq C\left\|\frac{\partial \widetilde{E}_{T}^{\delta}}{\partial x_{3}}(\cdot, t)\right\|_{L^{2}(\Omega)} \leq C\left|J_{S}\right|_{1,1, T}
$$

where we have used the stability estimate (2.22). On the other hand, using separation of variables and the a priori estimate 3.1 , we also have

$$
\left\|\frac{\partial V}{\partial x_{3}}(\cdot, t) \nabla \varphi_{S}^{\infty}\right\|_{L^{2}(\Omega)} \leq C\left|J_{S}\right|_{1,1, T}
$$


Finally, by $(4.31,4.32)$ and the triangular inequality, we get

$$
\left\|\frac{\partial v^{\delta}}{\partial x_{3}}(\cdot, t) \nabla \varphi_{S}^{\infty}\right\|_{L^{2}(\Omega)} \leq C\left|J_{S}\right|_{1,1, T}
$$

In the same way, one has

$$
\left\|\frac{\partial i^{\delta}}{\partial x_{3}}(\cdot, t) \nabla \psi_{s}^{\infty}\right\|_{L^{2}(\Omega)} \leq C\left|J_{S}\right|_{1,1, T}
$$

Then, substituting (4.33), (4.34) and (4.19) into (4.30), we get

$$
\frac{d}{d t} \mathcal{E}^{\delta} \leq C \delta \leq C \delta\left|J_{S}\right|_{1,1, T}^{2}
$$

which yields

$$
\left\|v^{\delta}\right\|_{L^{\infty}\left(0, T ; L^{2}(\mathbb{R})\right)}+\left\|i^{\delta}\right\|_{L^{\infty}\left(0, T ; L^{2}(\mathbb{R})\right)} \leq C(\delta T)^{\frac{1}{2}}\left|J_{S}\right|_{1,1, T} .
$$

Finally, the error estimate (3.14) is obtained by regrouping (4.14), (4.19) and (4.35).

Step 6 : proof of the error estimate (3.15).

To improve the estimate (4.35) when more regularity is assumed for the source term, we restart from (4.28) but do not integrate by part the right hand side. Instead, we apply directly Cauchy-Schwartz inequality to obtain

$$
\frac{1}{2} \frac{d}{d t} \mathcal{E}^{\delta} \leq C\left(\mathcal{E}^{\delta}\right)^{\frac{1}{2}}\left(\left\|\frac{\partial \widetilde{E}_{T}^{R, \delta}}{\partial x_{3}}\right\|_{L^{2}(\Omega)}+\left\|\frac{\partial \widetilde{H}_{T}^{R, \delta}}{\partial x_{3}}\right\|_{L^{2}(\Omega)}\right)
$$

which gives, after using Gronwall's lemma:

$$
\mathcal{E}^{\delta}(t)^{\frac{1}{2}} \leq C\left(\left\|\frac{\partial \widetilde{E}_{T}^{R, \delta}}{\partial x_{3}}\right\|_{L^{1}\left(0, T ; L^{2}(\Omega)\right)}+\left\|\frac{\partial \widetilde{H}_{T}^{R, \delta}}{\partial x_{3}}\right\|_{L^{1}\left(0, T ; L^{2}(\Omega)\right)}\right) .
$$

Then, if $J_{S} \in W_{0}^{2}(\Omega \times[0, T])$, we can use the inequality (4.20) to get

$$
\mathcal{E}^{\delta}(t)^{\frac{1}{2}} \leq C \delta\left(\left|J_{S}\right|_{2,1, T}\right)
$$

and if $J_{S} \in W_{0}^{3}(\Omega \times[0, T])$ we can use (4.22) to obtain

$$
\mathcal{E}^{\delta}(t)^{\frac{1}{2}} \leq C \delta^{2}\left(\left|J_{S}\right|_{3,1, T}+\left|J_{S}\right|_{2,2, T}\right)
$$




\section{Appendix}

In this section, we prove the proposition 4.1. The proposition 4.2 may be proven in a very similar way. We recall the proposition:

Proposition. There exist $C>0$ depending only on $(S, \varepsilon)$ such that,

$$
\forall u \in W(\varepsilon), \quad\|u\|_{L^{2}(S)} \leq C\left(\|\operatorname{rot} u\|_{L^{2}(S)}+\|\operatorname{div} \varepsilon u\|_{L^{2}(S)}+\left|\left(u, \nabla \varphi_{S}\right)_{\varepsilon}\right|\right) .
$$

The proof will be done in a classical way using contradiction arguments and the compactness properties proven in ( [1], [7]):

Property 5.1. $W(\varepsilon)$ (as defined by (4.6) ) is compactly embedded in $L^{2}(S)^{2}$.

Proof Assuming the proposition 4.1 is not true, we can construct a sequence $\left\{u_{n}\right\}$ such that

$$
\left\|\operatorname{rot} u_{n}\right\|_{L^{2}(S)}+\left\|\operatorname{div} \varepsilon u_{n}\right\|_{L^{2}(S)}+\left|\left(u_{n}, \nabla \varphi_{S}\right)_{\varepsilon}\right| \leq \frac{1}{n},
$$

and

$$
\left\|u_{n}\right\|_{L^{2}(S)}=1, \quad u_{n} \times n=0 .
$$

From the compactness property of $W(\varepsilon)$, we know that there exist $u \in W(\varepsilon)$ such that

$$
u_{n} \rightarrow u \quad \text { in } L^{2}(S)^{2} \quad \text { with } \quad \operatorname{rot} u=\operatorname{div} \varepsilon u=0, \quad\left(\varepsilon u, \nabla \varphi_{s}\right)_{L^{2}(S)}=0,
$$

and the limit $u$ also satisfy

$$
\|u\|_{L^{2}(S)}=1, \quad u \times n=0 .
$$

From the equation (5.1) and the definition of $U(\varepsilon)$ given by (4.6), we know that $u \in$ $U(\varepsilon)^{\perp}$ which means, from the decomposition property (4.5) $: u=\alpha_{u} \nabla \varphi_{s}$, with $\alpha_{u} \in \mathbb{R}$. And so

$$
\left(u, \nabla \varphi_{s}\right)_{\varepsilon}=0 \Rightarrow \alpha_{u}=0 \Rightarrow u=0,
$$

which contradicts the fact that we expected $\|u\|_{L^{2}(S)}=1$.

\section{References}

[1] S Caorsi, P Fernandes, and M Raffetto. On the convergence of Galerkin finite element approximations of electromagnetic eigenproblems. SIAM Journal on Numerical Analysis, 38(2):580-607 (electronic), 2000.

[2] R Dautray and J L Lions. Mathematical analysis and numerical methods for science and technology. Vol. 3. Springer-Verlag, 1990.

[3] D A Hill and J R Wait. Propagation Along a Coaxial-Cable with a Helical Shield. Ieee Transactions on Microwave Theory and Techniques, 28(2):84-89, 1980. 
[4] S Imperiale and P Joly. Mathematical modeling of electromagnetic wave propagation in heterogeneous lossy coaxial cables with variable cross section. (submitted), pages 1-25.

[5] S Imperiale and P Joly. Mathematical and numerical modelling of piezoelectric sensors. ESAIM: Mathematical Modelling and Numerical Analysis, (46):875-909, July 2012.

[6] P Joly. Variational Methods for Time Dependant Wave Propagation. In M Ainsworth, P Davies, D Duncan, P Martin, and B Rynne, editors, Topics in computational wave propagation: Direct and Inverse Problems. Computational Methods in Wave Propagation, pages 201-264. Springer Verlag, October 2003.

[7] P Monk. Finite element methods for Maxwell's equations. Oxford science publications, 2003.

[8] C Rose and M J Gans. A Dielectric-Free Superconducting Coaxial-Cable. Ieee Transactions on Microwave Theory and Techniques, 38(2):166-177, 1990.

[9] L W Schmerr Jr and S J Song. Ultrasonic Nondestructive Evaluation Systems. Springer, 2007. 